\title{
Benzyl alcohol-ammonia (1:1) cluster structure investigated by combined IR-UV double resonance spectroscopy in jet and $a b$ initio calculation
}

\author{
NIKHIL GUCHHAIT \\ Department of Chemistry, Indian Institute of Technology, Guwahati 781 039, \\ India \\ e-mail: nikhil@iitg.ernet.net
}

MS received 30 January 2001; revised 29 March 2001

\begin{abstract}
Laser-induced fluorescence excitation and IR-UV double resonance spectroscopy have been used to determine the hydrogen-bonded structure of benzyl alcohol-ammonia (1:1) cluster in a jet-cooled molecular beam. In addition, ab initio quantum chemical calculations have been performed at HF/6-31 $G$ and $\mathrm{HF} / 6-31 G(d, p)$ levels for different ground state equilibrium structures of the cluster to correlate the calculated $\mathrm{OH}$ and $\mathrm{NH}$ frequencies and their intensities with experimental results. The broad red-shifted $\mathrm{OH}$-stretching mode in the IR-UV double resonance spectrum suggests strong hydrogen bonding between the hydroxyl hydrogen and the lone pair of the ammonia nitrogen. The position and intensity distribution of the calculated $\mathrm{NH}$ and $\mathrm{OH}$ modes for the minimum-energy gauche form at HF/6-31G level have better correlation with the experimental results compared to other calculated ground state equilibrium conformers. These results lead to the conclusion that the minimum energy gauche form of the cluster is populated in the jet-cooled condition.
\end{abstract}

Keywords. Benzyl alcohol-ammonia cluster; IR-UV double resonance; ab-initio calculations; jet-cooled molecular beams.

\section{Introduction}

In recent years, hydrogen-bonded clusters of aromatic molecules with various solvents have received the attention of researchers to study the microscopic properties of solutions and biological environments ${ }^{1-12}$. Gas phase vibration spectroscopies like IR-UV and stimulated Raman-UV double resonance have been used for characterization of cluster structure and tunneling dynamics of hydrogen atom(s) ${ }^{13-18}$. It has been found that $\mathrm{OH}-$ stretching vibrations are sensitive probes for obtaining information on hydrogen bond and proton transfer phenomena in clusters. Mikami et al investigated $\mathrm{OH}, \mathrm{NH}$ and $\mathrm{CH}$ stretching frequencies by IR-UV double resonance spectroscopy of phenol and $\beta$ naphthol with a variety of amines, and predicted the non-proton-transferred structures of the clusters ${ }^{15,19}$. They found a large red-shift of the OH-stretching mode of the phenol and naphthol moieties in the clusters compared to the bare molecule which reflected a strong proton affinity of the amine group in the clusters.

The molecule benzyl alcohol is of particular interest as it has a vast synthetic utility, found in the natural product, and is also useful for mechanistic investigation ${ }^{20-22}$. Although it is simply a phenyl substituted methanol, the presence of $\pi$-electrons and a 
flexible tetrahedral carbon centre in the side chain is the main cause for the formation of several ground state equilibrium geometries of gauche, planar and perpendicular forms. A number of experimental and theoretical works have been carried out to resolve the longterm structural ambiguity of benzyl alcohol ${ }^{23-25}$. Recently, we performed fluorescence detected infrared (FDIR) spectroscopy in jet and carried out $a b$ initio calculation for benzyl alcohol and its water cluster ${ }^{26,27}$. Very recently, an independent IR-UV ion dip spectroscopy and high level $a b$ initio calculation were also carried out by Simons's group ${ }^{28}$. They showed that only the gauche conformer which has a small red shifted $\mathrm{OH}$ stretching mode due to weak hydrogen bonding with the benzene $\pi$-electron is populated in the jet-cooled condition.

In this paper, the structure of benzyl alcohol-ammonia (1:1) adduct has been investigated in the jets. Laser-induced fluorescence excitation (LIF) and fluorescence detected IR (FDIR) spectroscopic methods have been used to observe the $\mathrm{OH}-$ and $\mathrm{NH}-$ stretching modes. In addition, ab initio calculation at $\mathrm{HF} / 6-31 G$ and $\mathrm{HF} / 6-31 G(d, p)$ levels have been carried out to predict the probable ground state equilibrium structure and compare the simulated spectrum with the experimental one.

\section{Experimental procedures}

The experimental details of laser-induced fluorescence (LIF) excitation and IR-UV double resonance spectroscopic measurement have been described earlier ${ }^{12,18}$. Heated sample $(330 \mathrm{~K})$ vapour with helium carrier gas is expanded through a pulse nozzle to get the jet-cooled molecules. Stagnation pressure of $\mathrm{He}$ is $\sim 2 \mathrm{~atm}$, where the typical background pressure of the vacuum chamber is $1 \times 10^{-5}$ Torr. In the measurement of the LIF spectrum, the jet-cooled molecules are excited with a tunable UV light generated by a second harmonic of a $\mathrm{XeCl}$ excimer laser pumped dye laser (Lambda Physik LPX 100/FL 2002), and the total fluorescence is detected by a photomultiplier tube (Hamamatsu Photonics IP 28). The output signal is processed by a boxcar integrator (PAR 4420), and finally stored in a personal computer.

For fluorescence-detected infrared (FDIR) spectroscopy, the ground state population of a particular species in the jet is monitored by the fluorescence intensity excited by an UV light through the $S_{1}$ state. The IR pulse is introduced $\sim 50 \mathrm{~ns}$ prior to the UV pulse. When the IR frequency is resonant to the vibrational transition, the ground state population is reduced, resulting in a depletion of the fluorescence signal. Thus, the fluorescence-dip spectrum, or the so-called fluorescence detected IR spectrum (FDIR), is obtained. The second harmonic of a Nd:YAG laser (Quanta Ray GCR 230) and the output of the dye laser (continuum ND-6000) pumped by the same ND-YAG laser, are used to generate tunable IR light with a $\mathrm{LiNbO}_{3}$ crystal by difference frequency mixing method. The tunable IR output is passed through a $\mathrm{CaF}_{2}$ Brewster-angle window and dichroic mirrors to eliminate the visible light. Both the UV and IR beams are introduced into the vacuum chamber in a counter-propagating fashion and coaxially focused by lenses $(f=250 \mathrm{~mm}$ for IR and $f=500$ for $\mathrm{UV}$ ) on the supersonic jet. The fluorescence is detected by a photomultiplier and the photocurrent is processed by the boxcar integrator, and finally accumulated by a computer.

Benzyl alcohol was purchased from Aldrich Chemical Co. and was used without further purification. Ammonia ( 1\% of total pressure) was mixed with the helium carrier gas in a separate chamber and the mixture was expanded with the heated sample to make benzyl alcohol ammonia clusters. 
$A b$ initio quantum chemical calculations for obtaining different equilibrium structures of benzyl alcohol-ammonia cluster were carried out using the Gaussian 94 program at the $\mathrm{HF} / 6-31 G$ and HF/6-31G $(d, p)$ levels ${ }^{29}$. Calculated parameters are presented in $\$ 4$ in tabulated form. Simulated spectra for $\mathrm{OH}-$ and $\mathrm{NH}$-stretching frequencies and intensities are compared with the experimental spectrum.

\section{Results and discussions}

The LIF spectrum of benzyl alcohol is shown in figure 1a. Our LIF spectrum is more or less similar to that of the spectrum reported by Bernstein $e \mathrm{al}^{25}$. A new band is observed at $-46 \mathrm{~cm}^{-1}$ with respect to the strongest band at $37527 \mathrm{~cm}^{-1}$. This new band is also observed by Simons's group ${ }^{28}$. The calculated $(\mathrm{HF} / 6-31 G)$ low energy conformers of benzyl alcohol are shown in figure 2. In the minimum energy gauche form (conformer I), the hydrogen of $\mathrm{OH}$ group is directed towards the benzene $\pi$-electrons and has the ability to form weak $\pi$-electron hydrogen bonds. Conformer II is a relatively high energy conformer. In this conformer, the hydrogen of $\mathrm{OH}$ is in the plane of the ring and thus there is no possibility of hydrogen bonding. In our IR-UV double resonance spectroscopic study, the dominant band (i.e. $37527 \mathrm{~cm}^{-1}$ in the LIF spectrum) shows OHstretching frequency at $3650 \mathrm{~cm}^{-1}$. Simons et al assigned this band to the gauche conformer having slightly red-shifted $\mathrm{OH}$ frequency owing to the $\pi$-hydrogen bond ${ }^{28}$. It

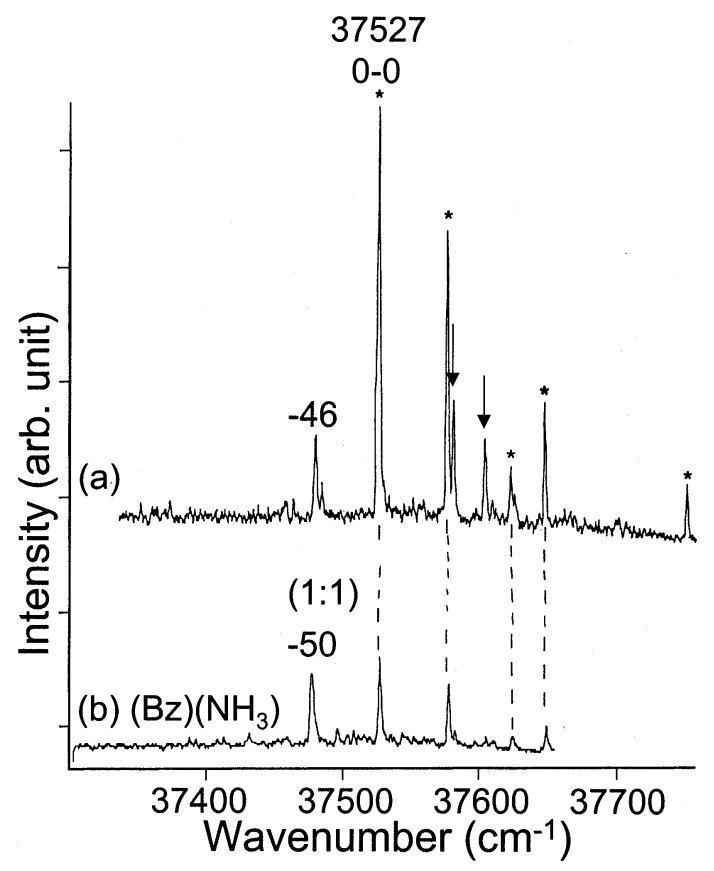

Figure 1. Laser-induced fluorescence (LIF) excitation spectra of jet-cooled benzyl alcohol and its ammonia cluster in the band origin region for the $S_{1}-S_{0}$ transition. (a) Benzyl alcohol, (b) cluster with ammonia. The bands marked by asterisk in (a) are the vibronic bands belonging to one conformer. Bands marked by arrows are due to water clusters ${ }^{27}$. 


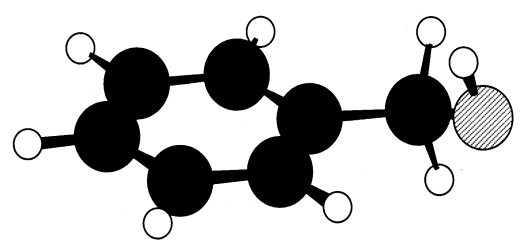

Conformer I $\left(0.0 \mathrm{~cm}^{-1}\right)$

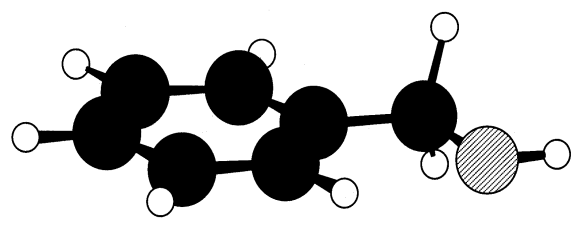

Conformer II $\left(+482 \mathrm{~cm}^{-1}\right)$

Figure 2. Ab initio (HF/6-31G) calculated equilibrium structures of benzyl alcohol with their relative energies. Conformer I: minimum energy gauche structure; conformer II: planar structure.

was also seen that all the bands on the blue side, marked by asterisks in figure 1a, have the same OH-stretching frequency of $3650 \mathrm{~cm}^{-126}$. This clearly indicates that all these bands arise from the same conformer and that all blue-side bands are nothing but the lowfrequency torsional mode of the hydroxy alkyl group as was interpreted by Bernstein $e t$ $a l^{25}$. In our measurement, the much weaker band in the excitation spectrum at $-46 \mathrm{~cm}^{-1}$ shows red-shifted OH-stretching frequency at $3585 \mathrm{~cm}^{-1}$. However, Simons's group observed resonance two-photon ionization (R2PI) and confirmed that $-46 \mathrm{~cm}^{-1}$ band is due to the benzyl alcohol dimmer ${ }^{28}$. In their study, $-46 \mathrm{~cm}^{-1}$ band appears both in the dimer and monomer mass channel. They also found two $\mathrm{OH}$-stretching frequency of 3515 and $3584 \mathrm{~cm}^{-1}$ in the IR-UV dip spectrum for the $-46 \mathrm{~cm}^{-1}$ band. This firmly supports their assignment of $-46 \mathrm{~cm}^{-1}$ band being due to dimer. By high level $a b$ initio calculations, they showed that two gauche forms of monomer make $\sigma$ - and $\pi$-type hydrogen bond to form the dimer.

The LIF spectrum of benzyl alcohol-ammonia (1:1) cluster is shown in figure $1 \mathrm{~b}$, where the origin band for the cluster appears at $-50 \mathrm{~cm}^{-1}$ with respect to the $0-0$ band $\left(37527 \mathrm{~cm}^{-1}\right)$ of the bare benzyl alcohol molecule. Earlier, Bernstein's group investigated ion fragmentation chemistry of benzyl alcohol ammonia clusters by mass-resolved excitation study in jet ${ }^{30}$. Our observed $0-0$ band of the (1:1) cluster has good agreement with their reported spectrum. For the measurement of FDIR spectrum of the cluster, the UV light was fixed at $-50 \mathrm{~cm}^{-1}$ band and the IR light was scanned. Figure $3 \mathrm{a}$ shows the FDIR spectrum of benzyl alcohol-ammonia (1:1) cluster where an intense band and two weak bands are observed at 3406, 3432 and $3441 \mathrm{~cm}^{-1}$ respectively. The observed IR frequency and intensity are presented in table 1 . Since $\mathrm{OH}$-stretching vibrations are known to be generally intense in the IR spectra, the broad and intense band at $3406 \mathrm{~cm}^{-1}$ can be assigned to the $\mathrm{OH}$-stretching vibrations, which exhibit a substantial red-shift of $244 \mathrm{~cm}^{-1}$ from the OH-stretching mode of bare benzyl alcohol ${ }^{26}$. Such a large red-shift indicates strong hydrogen bonding between the alcoholic $\mathrm{OH}$ and the nitrogen lone pair. 


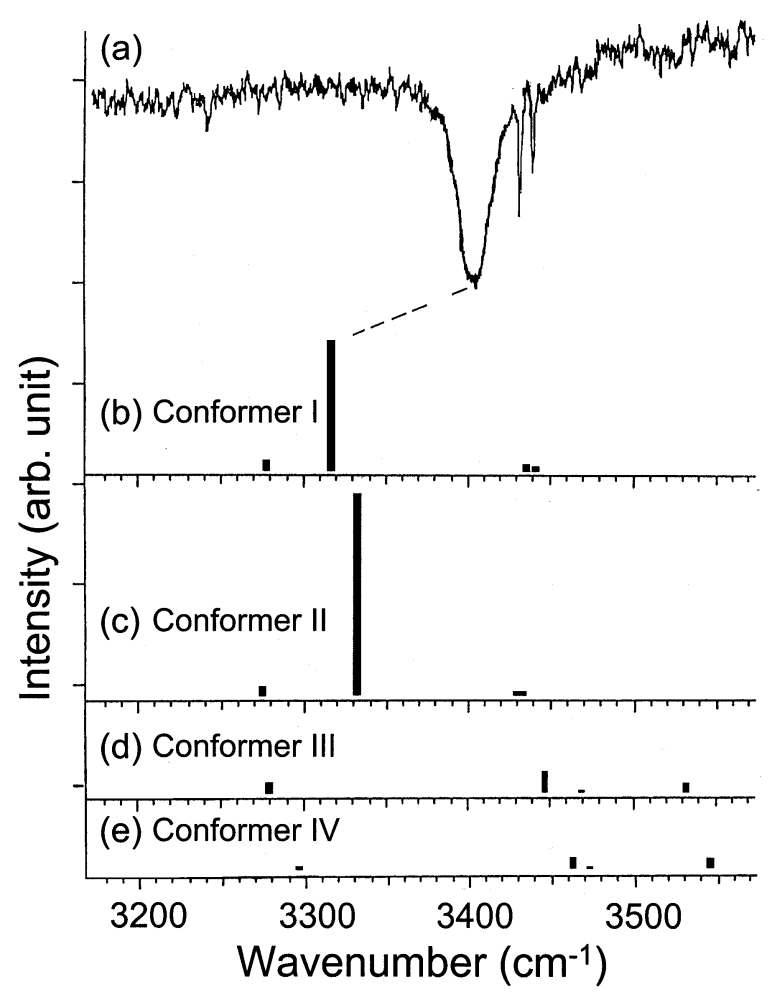

Figure 3. (a) The observed FDIR spectrum of the OH- and NH-stretching bands of benzyl alcohol-ammonia cluster (1:1). Calculated spectra (stick diagram) of four conformers at the HF/6-31G level. (b) Conformer I; (c) conformer II; (d) conformer III; and (e) conformer IV. The height of the bar represents the integrated absorption intensity.

The red-shift of the $\mathrm{OH}$ mode in phenol ammonia and $\beta$-naphthol ammonia (1:1) clusters is about 363 and $\sim 400 \mathrm{~cm}^{-1}$ respectively ${ }^{15,19}$. However, in benzyl alcohol this red-shift is comparatively small in comparison with the phenol ammonia or $\beta$-naphthol ammonia clusters, which means that the hydrogen bond strength is weaker compared to the phenol or $\beta$-naphthol ammonia system. This is presumably due to weak acidic property of benzyl alcohol compared to phenol or $\beta$-naphthol. Although all the normal modes of ammonia are IR active, but the IR intensity of asymmetric modes is higher than the symmetric mode. It is also evident that symmetric mode at the ammonia site is weaker in the IR transition upon cluster formation in phenol- $\left(\mathrm{NH}_{3}\right)_{1,2}$ clusters ${ }^{19,31}$. Therefore, two other weak bands at 3432 and $3441 \mathrm{~cm}^{-1}$ can be assigned to the $v_{3}$ (asymmetric) modes of the ammonia site. The absence of $v_{1}$ (symmetric) mode in our spectrum may be due its very weak intensity. Our calculations show that the intensity of $v_{1}$ mode is stronger than $v_{3}$ modes at HF/6-31G level but weaker at HF/6-31G(d,p) level. This indicates that at high level calculations may provide a better agreement of intensity distribution than the low level calculation for the cluster. The 3432 and $3441 \mathrm{~cm}^{-1}$ bands exhibit a frequency shift of 5 and $12 \mathrm{~cm}^{-1}$ compared to the $\mathrm{NH}$ modes of free ammonia ${ }^{15}$. The $\mathrm{v}_{3}$ mode is degenerate in pure ammonia. The splitting of $\mathrm{NH}$ modes in the clusters indicates that 
Table 1. Observed vibrational modes and ab initio calculated frequencies at HF/6$31 G$ level for benzyl alcohol and its (1:1) ammonia cluster.

\begin{tabular}{|c|c|c|c|c|c|}
\hline \multirow[b]{2}{*}{ Cluster size } & \multicolumn{3}{|c|}{ Observed data } & \multicolumn{2}{|c|}{ Calculated data } \\
\hline & $\begin{array}{c}\text { Frequency } \\
\left(\mathrm{cm}^{-1}\right)\end{array}$ & Intensity & Assignment & $\begin{array}{l}\text { Frequency } \\
\left(\mathrm{cm}^{-1}\right)^{\mathrm{a}}\end{array}$ & Assignment \\
\hline$n=0$ & 3650 & $S$ & $v^{\mathrm{OH}}$ & $\begin{array}{l}3529(40) \\
3556(29)\end{array}$ & $v^{\mathrm{OH}}$ \\
\hline$n=1$ & $\begin{array}{l}3441 \\
3432\end{array}$ & $\begin{array}{l}W \\
M\end{array}$ & $\begin{array}{l}v_{3}{ }^{\mathrm{NH}} \\
v_{3}{ }^{N H}\end{array}$ & $\begin{array}{l}3441(18) \\
3437(21), \\
3278(38)\end{array}$ & $\begin{array}{l}v_{3}^{\mathrm{NH}} \\
v_{3}{ }^{\mathrm{NH}} \\
v_{1}^{\mathrm{NH}} \\
v_{\mathrm{OH}}^{\mathrm{OH}}\end{array}$ \\
\hline
\end{tabular}

${ }^{\mathrm{a} C}$ Calculated $\mathrm{OH}$ and $\mathrm{NH}$ frequencies are multiplied by a factor of 0.8795 for benzyl alcohol and its ammonia 1:1 cluster (conformer I of figure 4). IR intensities $(\mathrm{km} / \mathrm{Mol})$ are shown in parentheses. All values are rounded off to the nearest number

${ }^{\mathrm{b}}$ Calculated frequency and IR intensity of gauche (conformer I) and planar forms (conformer II) respectively ${ }^{26}$.

three ammonium hydrogens are not equivalent in the cluster. Weak NH bands with a lifting of degeneracy of $v_{3}$ mode implies the reduction of $C_{3}$ symmetry in the cluster. A weak perturbation by $\pi$-bond may be the cause for this type of behaviour.

Figure 4 shows four possible low energy equilibrium conformers of benzyl alcoholammonia (1:1) cluster obtained by ab initio calculations at the HF/6-31G or HF/6-31G(d, $p$ ) level. In the present case the level of calculation is not high enough but the trend in relative stability of the conformers at both levels is the same. In the minimum energy conformer I, the alcoholic hydrogen forms a hydrogen bond with the lone pair of nitrogen, and ammonia is placed in such a way that one of the hydrogens is directed towards the benzene ring, and this probably forms a weak $\pi$-type hydrogen bond. In this conformer, the three hydrogens of ammonia are not equivalent. The second lower energy conformer II is a planar one and has no chance of forming a $\pi$-type hydrogen bond, and the binding nature is similar to that of phenol or $\beta$-naphthol ammonia clusters. In this conformer the three hydrogens are more or less equivalent. In two other higher energy conformers (III and IV), ammonia acts as a proton donor, where the hydrogen of the $\mathrm{OH}$ group is not involved in hydrogen bonding with the lone pair of the nitrogen.

The calculated $\mathrm{OH}$ and $\mathrm{NH}$ frequencies and their intensities at both HF/6-31G and $\mathrm{HF} / 6-31 G(d, p)$ levels for all the conformers are shown in table 2 . To correlate the calculated frequencies with the experimental results, the calculated frequencies are multiplied by a factor of 0.8795 and 0.8610 for $\mathrm{HF} / 6-31 G$ and $\mathrm{HF} / 6-31 G(d, p)$ levels respectively. It might be possible to improve the multiplication factor and relative intensity using high level calculations such as the DFT method. The calculated OH mode is at lower frequency than those of NH modes with the HF/6-31G basis set. It is expected that the nature of the hydrogen-bonded $\mathrm{OH}$ mode should be predicted more successfully with lager basis set $(\mathrm{HF} / 6-31 G(d, p))$ than the $\mathrm{HF} / 6-31 G$ basis set calculation. But the $\mathrm{OH}$-stretching mode is at much higher frequency than those of the NH modes with HF/6$31 G(d, p)$ calculation (table 2). Very similar results were found in case of phenol ammonia and $\beta$-naphthol ammonia clusters ${ }^{15,19}$. Such a basis set dependence of calculated vibrational frequencies has been reported by Schutz et $a l^{32}$ for the phenol 


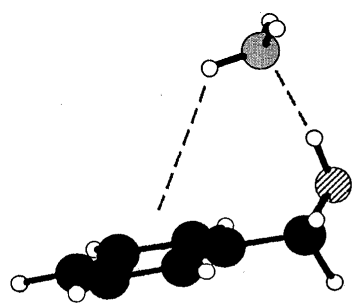

Conformer I
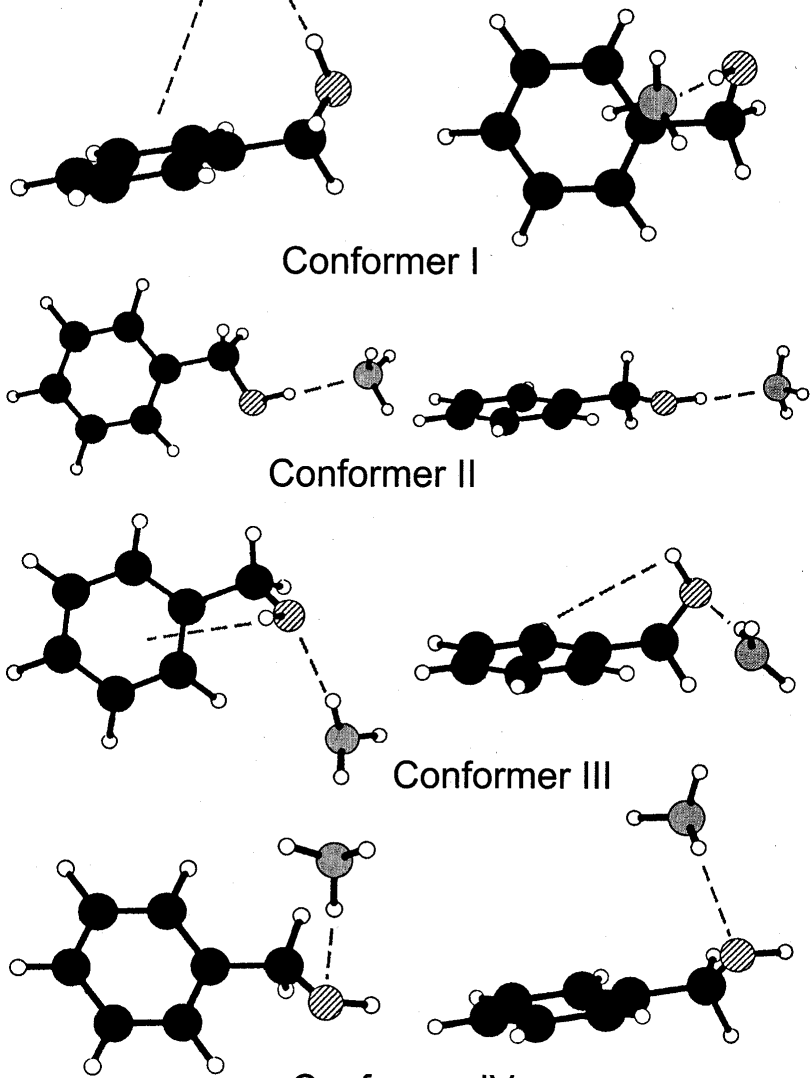

Conformer IV

Figure 4. Energy optimized structures for benzyl alcohol-ammonia (1:1) cluster at the HF/6-31G level. Conformer I: minimum energy gauche type ring structure; conformer II: planar type conformer; conformer III: higher energy gauche type conformer; and conformer IV: higher energy planar type conformer. Possible hydrogen bonding is shown by broken lines.

water cluster. Accidental agreement between the observed IR spectrum with the calculated results at $\mathrm{HF} / 6-31 G$ level is observed. The simulated spectra at $\mathrm{HF} / 6-31 G$ level for conformers I to IV are shown in figures $3 \mathrm{~b}$ to $3 \mathrm{e}$ respectively. The possibility of observing conformers III and IV can be easily eliminated as follows; (i) their bands appear on the higher frequency side; (ii) their intensity distributions are totally different from that of the observed FDIR spectrum; (iii) these are comparatively higher energy conformers and we can expect that these will not be populated in our jet-cooled condition; (iv) since ammonia behaves as a proton donor the $\mathrm{OH}$ frequency will not be red-shifted. For conformers I and II, the simulated spectra are similar to each other and correspond well to the observed spectrum. Their energy difference is also not large enough. However, it is seen that the gauche conformer I is the minimum energy form at 
Table 2. Calculated energies, IR frequencies and intensities at the HF/6-31G and HF/6-31G $(d, p)$ levels of benzyl alcohol-ammonia (1:1) cluster ${ }^{\mathrm{a}, \mathrm{b}}$.

\begin{tabular}{lllll}
\hline Structure & $\mathrm{HF} / 6-31 G$ & Assignment $^{\mathrm{c}}$ & $\mathrm{HF} / 6-31 G(d, p)$ & Assignment $^{\mathrm{c}}$ \\
\hline
\end{tabular}

Conformer I

$\Delta E\left(\mathrm{~cm}^{-1}\right)^{\mathrm{d}}$ 0 $3441^{\mathrm{e}}(18)$ $3437(21)$

$3317(480)$

$3278(38)$

$$
\begin{aligned}
& v_{3}{ }^{\mathrm{NH}} \\
& v_{3}{ }^{\mathrm{NH}} \\
& v^{\mathrm{OH}} \\
& v_{1}{ }^{\mathrm{NH}}
\end{aligned}
$$

$3441^{\mathrm{e}}(437)$

$3309(7)$

$3308(8)$

$3190(2)$

$$
+515
$$$$
\text { 3459(683) }
$$

3308(7)

3306(8)

3190(6)

$v_{3}{ }^{\mathrm{NH}}$
$v_{3}{ }^{\mathrm{NH}}$
$v^{\mathrm{OH}}$
$v_{1}{ }^{\mathrm{NH}}$

$\mathrm{v}_{1}{ }^{\mathrm{NH}}$

Conformer III

$\Delta E\left(\mathrm{~cm}^{-1}\right)^{\mathrm{d}}$

$$
\begin{aligned}
& +1332 \\
& 3532(32) \\
& 3469(7) \\
& 3447(78) \\
& 3280(41)
\end{aligned}
$$

$$
\begin{aligned}
& v^{\mathrm{OH}} \\
& v_{3}{ }^{\mathrm{NH}} \\
& v_{3}{ }^{\mathrm{NH}} \\
& v_{1}{ }^{\mathrm{NH}}
\end{aligned}
$$

Conformer II

3333(750)

$+1334$

3593(46)

3303(0.5)

$3300(31)$

3182(17)

$$
\begin{aligned}
& v^{\mathrm{OH}} \\
& v^{\mathrm{NH}} \\
& v^{\mathrm{NH}} \\
& v_{1}{ }^{\mathrm{NH}}
\end{aligned}
$$

Conformer IV

\begin{tabular}{lllll}
$\Delta E\left(\mathrm{~cm}^{-1}\right)^{\mathrm{d}}$ & +2106 & & +1942 & \\
& $3546(40)$ & $v_{3}{ }^{\mathrm{NH}}$ & $3605(62)$ & $v^{\mathrm{OH}}$ \\
$3473(7)$ & $v_{3}{ }^{\mathrm{NH}}$ & $3304(12)$ & $\mathrm{v}^{\mathrm{NH}}$ \\
& $3463(46)$ & $v^{\mathrm{OH}}$ & $3302(3)$ & $\mathrm{v}^{\mathrm{NH}}$ \\
& $3296(14)$ & $\mathrm{v}_{1}{ }^{\mathrm{NH}}$ & $3186(8)$ & $\mathrm{v}_{1}{ }^{\mathrm{NH}}$ \\
\hline
\end{tabular}

${ }^{a}$ All values are given in $\mathrm{cm}^{-1}$ and rounded off up to the nearest whole number.

${ }^{\mathrm{b}}$ Calculated frequencies are multiplied by factors of 0.8795 and 0.8610 for HF/6-31G and $\mathrm{HF} / 6-31 G(d, p)$ levels respectively. Numbers in parentheses are calculated IR intensities

${ }^{\mathrm{c}}$ Assignments of calculated normal modes

${ }^{\mathrm{d}}$ Relative energy with respect to conformer I

${ }^{\mathrm{e}}$ Frequencies in $\mathrm{cm}^{-1}$

${ }^{\mathrm{f}} \mathrm{IR}$ intensity in $\mathrm{km} / \mathrm{Mol}$.

both levels of calculation. The $\sigma$ - and $\pi$-type hydrogen bonds of conformer I may provide extra stabilization compared to the planar conformer II which has only $\sigma$-type hydrogen bonds. Second, it was seen earlier ${ }^{26-28}$ that only the low energy gauche form of bare benzyl alcohol, its dimer and hydrogen-bonded water clusters are populated in the jet. Thus we can expect that, during the process of jet cooling, all higher energy conformers try to freeze to the minimum energy gauche form. The minimum energy gauche form of the ammonia cluster (conformer I) may therefore be attributed to the observed cluster in jet. Third, it is seen that the structure of phenol ammonia $(1: 1)$ cluster is similar to that of 
conformer II of the benzyl alcohol-ammonia (1:1) cluster ${ }^{15}$. If the FDIR spectrum corresponds to Conformer II, we should expect similar spectra as was observed in the case of the phenol ammonia (1:1) cluster where only one weak NH band was observed. But here, the FDIR spectrum is of different from that of phenol ammonia (1:1) clusters. The shifting of degeneracy of $v_{3}$ modes in the cluster provides clear evidence for the existence of a gauche-form cluster. Based on all these points we can say that the spectrum of benzyl alcohol ammonia $(1: 1)$ cluster in the jet is due to the minimum energy gauche form (conformer I).

\section{Summary}

In this paper, LIF and IR-UV double resonance spectroscopies in jet have been used for looking at $\mathrm{OH}$ - and $\mathrm{NH}$-stretching frequencies and thus for determining the structure of the benzyl alcohol-ammonia (1:1) cluster. A large $244 \mathrm{~cm}^{-1}$ red-shifted OH-stretching vibration in the FDIR spectrum reflects strong hydrogen bonding between the hydroxyl hydrogen and the lone pair of nitrogen. Ab initio calculations by HF/6-31G and HF/6$31 G(d, p)$ levels have been done for different ground state equilibrium structures in order to correlate the simulated spectra of the $\mathrm{OH}$ and $\mathrm{NH}$ modes with the observed FDIR spectrum. Our results lead to the conclusion that the minimum energy gauche conformer is populated in the jet, where benzyl alcohol acts as a proton donor and ammonia acts as proton acceptor. More accurate $a b$ initio calculations are needed to get better correlation of experimental results with calculated values.

\section{Acknowledgements}

The author is grateful to the Japan Society for the Promotion of Science for a fellowship and also wishes to express his indebtedness to Professors N Mikami and T Ebata, Quantum Chemistry Lab, Tohoku University for allowing him to do this work at their lab. The author would like to express his sincere thanks to Prof. Mihir Chowdhury of The Indian Association for the Cultivation of Science, Calcutta for helpful discussions and to the referee for his/her valuable suggestions and comments.

\section{References}

1. Ebata T, Fujii A and Mikami N 1998 Int. Rev. Phy. Chem. 17331

2. Hockridge M R and Robertson E G 1999 J. Phys. Chem. A103 3618

3. Matsuda Y, Ebata T and Mikami N 1999 J. Chem. Phys. 1108397

4. Liu K, Brown M G and Saykally R J 1997 J. Phys. Chem. A101 8995, and references therein

5. Stanley R J and Castleman A W Jr. 1993 J. Chem. Phys. 98796

6. Hockridge M R, Knight S M, Robertson E G, Simons J P, McCombie J and Walker M 1999 Phys. Chem. Chem. Phys. 1 407, and references therein

7. Pribble R N and Zwier T S 1994 Science 26575

8. Matsumoto Y, Ebata T and Mikami T 1998 J. Chem. Phys. 1096303

9. Helm R M, Vogel H P and Neusser H J 1997 J. Chem. Phys. 1084496

10. Schmitt M, Jacoby Ch and Kleinermanns K 1997 J. Chem. Phys. 1084486

11. Ebata T, Nagao K and Mikami N 1997 Chem. Phys. 231199

12. Ishikawa S, Ebata T, Tanabe S and Mikami N 1999 J. Chem. Phys. 1109504

13. Fujii A, Miyazaki M, Ebata T and Mikami N 1999 J. Chem. Phys. 11011125

14. Maxton P M, Schaeffer M W and Felker P M 1995 Chem. Phys. Lett. 241603

15. Matsumoto Y, Ebata T and Mikami N 2001 J. Mol. Struct. (in press)

16. Yamamoto R, Ishikawa S, Ebata T and Mikami N 2000 J. Raman Spectrosc. 31295 
17. Pribble R N and Zwier T S 1994 Faraday Discuss. 97229

18. Mikami N 1994 Bull. Chem. Soc. Jpn. 68683

19. Iwasaki A, Fujii A, Watanabe T, Ebata T and Mikami N 1996 J. Chem. Phys. 10016053

20. Knof S, Strassmair H, Engel J, Rothe M and Steffen K D 1972 Biopolymers 11731

21. Matheu M I, Echarri R and Castillon S 1993 Tetrahedron Lett. 342361

22. Faubel M, Steiner B and Toennies J P 1997 J. Chem. Phys. 1069013

23. Oki M and Iwamura H 1959 Bull. Chem. Soc. Jpn. 32950

24. Hehre W J, Radom L and Pople J A 1972 J. Am. Chem. Soc. 39 1496, and references therein

25. Im H S, Bernstein E R, Secor H V and Seeman J I 1991 J. Am. Chem. Soc. 1134422

26. Guchhait N, Ebata T and Mikami N 1999 J. Am. Chem. Soc. 1215705

27. Guchhait N, Ebata T and Mikami N 1999 J. Chem. Phys. 1118438

28. Mons M, Robertson E G and Simons J P 2000 J. Phys. Chem. A104 1430

29. Frisch M J et al 1995 Ab initio calculation program Gaussian 94 (Rev. D.4), Gaussian Inc., Pittsburgh, PA

30. Li S and Bernstein E R 1992 J. Chem. Phys. 977383

31. Schmitt M, Jacoby Ch, Gerhards M, Unterberg C, Roth W and Kleinermanns K 2000 J. Chem. Phys. 1132995

32. Schutz M, Burgi T and Leutwyler S 1992 J. Mol. Struct. (Theochem) 276117 